\title{
Utilización de medicina alternativa en mujeres con cáncer de mama
}

Use of alternative medicine by women with early-stage breast cance Burstein HJ, Gelber S, Guadagnoli et al. N Engl J Med 1999; 340:1733-9

\section{Objetivos}

Establecer la relación entre la terapia médica alternativa (TMA) y la convencional; así como los factores psicológicos y físicos, que contribuyen al uso de la medicina alternativa, en mujeres con cáncer de mama (CM) temprano.

\section{Diseño}

Estudio prospectivo de cohortes con seguimiento de un año.

Lugar

Hospitales seleccionados aleatorizadamente en Massachusetts, EE.UU.

\section{Pacientes}

Se incluyeron 657 mujeres con CM estadio I o II diagnosticadas entre 9/93 - 9/95. Las pacientes fueron contactadas telefónicamente por un investigador independiente, dentro de los 60 días de recibir tratamiento quirúrgico. Se obtuvo información del estadio y tipo de cirugía a través de la historia clínica.

\section{Medición de resultados principales}

Se realizó un cuestionario a los 3 y 12 meses luego de la cirugía, que incluía datos sociodemográficos, nivel educativo y económico, tratamientos médicos recibidos y sus efectos adversos. Se utilizaron instrumentos para medir el estado de salud físico y psíquico(SF 36 Short -Form Health Survey, altos valores en la escala significan mejor estado físico y emocional); la satisfacción sexual MOS (mayor puntaje significa mejor satisfacción sexual); síntomas de depresión CES-D (Center for Epidemiologic Studies, mayor puntaje más síntomas depresivos) y una lista de preguntas acerca de problemas relacionados con el CA, efectos de los distintos tratamientos, síntomas menopáusicos, y una escala (Lasry y Margolese) para evaluar el temor a la recurrencia del CA luego de la cirugía (a mayor puntaje más temor). Las pacientes completaron el SF 36 del mes previo a la cirugía en el cuestionario de los 3 meses y se lo utilizó como dato basal y contestaron acerca del uso de TMA durante ese año previo, en el cuestionario de los 12 meses. Para el análisis La TMA se clasificó en: terapias curativas (con acción en el cuerpo) como dietas, acupuntura, megavitaminas, etc; terapias psicológicas (que involucran procesos mentales) como hipnosis, biofeedback, relajación, etc.; TMA nueva (dentro del año después de la cirugía), continua (antes y después) o ninguna. Los pacientes que utilizaron TMA se agruparon en: no utilizadoras, y que alguna vez utilizaron (sea nueva o continua).

Se compararon los puntajes de los instrumentos entre nuevas utilizadoras de TMA y no utilizadoras y entre utilizadoras continuas y no utilizadoras; y se analizaron las diferencias entre el uso de TMA acorde al tratamiento convencional recibido y factores sociodemográficos.

\section{Resultados principales}

El 28.1\% de las 480 mujeres que completaron el cuestionario al año reportaron el uso de TMA. Las mujeres que respondieron $(n=480)$ tuvieron mayor nivel de educación e ingreso económico, la mayoría eran de raza blanca y tenían menos comorbilidades, vs. las que no respondieron $(p<0.001)$. Solo la edad joven y el alto nivel educativo estuvieron significativamente asociados al nuevo uso de TMA $(p<0.001)$. El nuevo uso de TMA no se asoció con el estadio del CA ni la terapia adyuvante recibida (controlado por otras variables).

\begin{tabular}{l|l|l|l|l|l|l|l}
\multicolumn{7}{c}{ Resultados significativos de uso de TMA y calidad de vida relacionada con la salud } \\
\hline & $\begin{array}{l}\text { No U } \\
(n=332)\end{array}$ & $\begin{array}{l}U c \\
(n=45)\end{array}$ & $\begin{array}{l}\text { NU } ~ \\
(n=90)\end{array}$ & $\begin{array}{l}\text { No U } \\
(n=332)\end{array}$ & $\begin{array}{l}\text { Uc } \\
(n=37)\end{array}$ & $\begin{array}{l}\text { NU } ~ \\
(n=103)\end{array}$ \\
\hline
\end{tabular}

æ No U: no utilizadoras Uc: utilizadoras continuo NU: nuevas utilizadoras $\# p<0.001$ comparando entre no No U y NU

Altos puntajes en la escala de temor a la recurrencia $(p=0.008)$, depresión (CES-D) $(p=0.09)$ y mayor nro. de síntomas $(p=0.01)$ fueron asociados al nuevo uso de terapias psicológicas. Todos los grupos reportaron mejoría en el puntaje de salud física y mental a los 12 meses de la cirugía, pero aquellas que comenzaron TMA reportaron menor satísfacción sexual y mayor temor a la recurrencia del cáncer.

\section{Conclusiones}

Entre las mujeres con diagnóstico reciente de cáncer de mama que han sido tratadas con terapias convencionales, el uso de TMA se asoció a peor calidad de vida y mayor dificultad psicosocial.

\section{COMENTARIO}

Diferentes tipos de técnicas complementarias o sustitutivas a las terapias médicas habituales han sido agrupadas en lo que llamamos "la medicina alternativa" y es actualmente conocido y difundido su uso en todo el mundo. La relación entre el uso de tratamientos médicos convencionales y alternativos no es claro. ¿Es una medicina "alternativa" porque representa un sustituto o un complemento de la medicina convencional?

Algunos estudios han tratado de establecer la asociación entre las características de ciertas poblaciones y la motivación para el uso de la medicina no convencional ${ }^{1-2}$ y una población particular son los pacientes con cáncer. Este estudio intenta investigar algunos factores que contribuyen al uso de terapias alternativas en una población de mujeres con diagnóstico reciente de CM. El $28 \%$ reportó el uso de TMA, pero es importante destacar que las mujeres participaban voluntariamente, tenían mejor nivel educativo y económico, y mejor estado físico. Probablemente estas mujeres no sean representativas de todas las mujeres con CM, limitando la validez externa del estudio*. El temor a la recurrencia, depresión y el mayor número de síntomas se asoció al nuevo uso de terapias psicológicas; lo cual sugiere que este grupo de pacientes busca tratamientos alternativos que consideren estos aspectos de su vida. Este parece ser un aporte importante del trabajo, en general, cerca de un tercio de los pacientes con cáncer refiere síntomas de ansiedad y depresión que frecuentemente no son detectados. ${ }^{3}$ En el presente estudio un $71 \%$ de las mujeres indicaron que sus médicos estaban de acuerdo con el uso de la terapia alternativa que utilizaban; esto parece ser un éxito en la relación médico- paciente y en la conocida barrera de rechazo entre lo convencional y alternativo para algunos grupos de la medicina.

Reconociendo las limitaciones de todo estudio descriptivo, este trabajo deja como aporte el haber investigado una vez más acerca de los factores psicológicos y la calidad de vida relacionada con la salud en pacientes con cáncer, y evalúa dónde y qué tipo de respuestas busca este tipo de pacientes. Una interesante editorial concluye: el principio básico de "nosotros tratamos a la persona, no solo la enfermedad" parece peligrar, cuando los pacientes, por cualquier causa, no nos hablan de su angustia o dolor y no esperan que nosotros la tratemos; esto es parte ineludible de los cuidados de la medicina convencional. ${ }^{4}$

1. Eisenberq DM, Kessler RC, Foster C, et al. Unconvetional medicine in the United States: prevalence, costs, and patterns of use. N Engl J Med 1993; $328: 246-52$.

2. Vickers A, Zollman C. ABC of complementary medicine BMJ 1999; 319 1050-1053, 101176-1179, 1346-49.

3. Downer SM, Cody MM, Mc Cluskey P et al. Pursuit and practices of complementary therapies by cancer patients receiving conventional treament BMJ 1994; 309:86-9 4. Use of alternative medicine, a marker for distress. N Engl J Med 1999; 340:1758-9 